\section{Mandibular Trabecular Bone Analysis Using Local Binary Pattern for Osteoporosis Diagnosis}

\author{
Khojastepour L. ${ }^{1}$, Hasani M. ${ }^{1}$, Ghasemi M..$^{1 *}$, Mehdizadeh A. \\ R. ${ }^{2}$, Tajeripour F. ${ }^{3}$
}

\begin{abstract}
Background: Osteoporosis is a systemic skeletal disease characterized by low bone mineral density (BMD) and micro-architectural deterioration of bone tissue, leading to bone fragility and increased fracture risk. Since Panoramic image is a feasible and relatively routine imaging technique in dentistry; it could provide an opportunistic chance for screening osteoporosis. In this regard, numerous panoramic derived indices have been developed and suggested for osteoporosis screening. Jaw trabecular pattern is one of the main bone strength factors and trabecular bone pattern assessment is important factor in bone quality analysis. Texture analysis applied to trabecular bone images offers an ability to exploit the information present on conventional radiographs.
\end{abstract}

Objective: The purpose of this study was to evaluate the relationship between Jaw trabecular pattern in panoramic image and osteoporosis based on image texture analyzing using local binary pattern.

Material and Methods: An experiment is evaluated in this paper based on a real hand-captured database of panoramic radiograph images from osteoporosis and non-osteoporosis person in Namazi Hospital, Shiraz, Iran .An approach is proposed for osteoporosis diagnosis consisting of two steps. First, modified version of local binary patterns is used to extract discriminative features from jaw panoramic radiograph images. Then, classification is done using different classifiers.

Results: Comparative results show that the proposed approach provides classification accuracy about $99.6 \%$, which is higher than many state-of-the-art methods.

Conclusion: High classification accuracy, low computational complexity, multiresolution and rotation invariant are among advantages of our proposed approach.

Keywords

Osteoporosis, Panoramic, Texture Analysis, Local Binary Pattern

\section{Introduction}

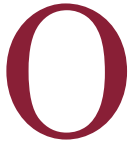

steoporosis is a systemic skeletal disease characterized by low bone mineral density (BMD) and micro-architectural deterioration of bone tissue, leading to bone fragility and increased fracture risk [1-4]. For the time being, DEXA is considered as a gold standard to measure BMD and osteoporosis [5-8]. Nonetheless, it is not widely available and is an expensive technique, so a less expensive alternative should be agreed upon to assess the skeletal status $[5,9,10]$. Because the panoramic radiography is an exam more common and affordable than
${ }^{1}$ Department of Oral and Maxillofacial Radiology,

School of Dentistry, Shi-

raz University of Medical

Sciences, Shiraz, Iran

${ }^{2}$ Department of Medical

Physics, School of Medi-

cine, Shiraz University of

Medical Sciences

${ }^{3}$ Department of Comput-

er Engineering, Science

and IT, Shiraz University,

Shiraz, Iran

Corresponding author:

M. Ghasemi

Postgraduate Student

of Oral and Maxillofacial

Radiology, Department

of Oral and Maxillofacial

Radiology, School of

Dentistry, Shiraz Univer-

sity of Medical Sciences,

Shiraz, Iran

E-mail: mghasemi411@

yahoo.com

Received: 26 February 2017

Accepted: 22 March 2017 
DEXA, and that it shows the entire maxillofacial region on a single film without requiring additional radiation exposure to the patient, its application in the early detection of low bone mass would bring a significant benefit of early treatment to those afflicted with osteoporosis [11, 12, 7, 13-15]. Horner et al. [8] concluded that mandibular BMD significantly correlated with bone density on other sites. In [16], Hidebolt claimed that dental x-ray is a highly effective method for distinguishing patients with osteoporosis from a patient with normal bone density.

Researchers have developed a number of panoramic radiomorphmetric indices, image processing and analyzing techniques for the quantification of mandibular bone mass and trabecular architecture to discriminate osteoporotic patients from non-osteoporotic ones [14]. These indices include cortical index (CI), mandibular cortical width (MCW), panoramic index (PMI) and alveolar crest resorption ratio $(\mathrm{M} \backslash \mathrm{M}$ Ratio). Several studies have been carried out in this regard, some of which have reported a positive relationship between mandibular radio morphometric indices and BMD $[8,9]$. On the other hand, some other studies have not reported any relationship between these indices and BMD $[17,18]$. Among these indices, MCI is relatively simple because no measurements or calculations are required but it depends on visual assessments, its repeatability has been evaluated especially interobserver agreement is reported to be poor in some studies [19]. However, some researchers have reported a satisfactory level of inter- and intra-observer agreements [20, 21, 9].

In order to reduce the subjectivity of observers, and according to the fact that BMD is not the only one contributing to bone strength, analyzing trabecular architecture is noticed to discriminate healthy and osteoporotic patients $[5,14]$.

Assessment of trabecular bone pattern is an important factor in the analysis of mandibular bone quality. Texture analysis applied to tra- becular bone images offers the ability to exploit the information present on conventional radiographs [22-24].

There are many algorithms to extract the feature of trabecular pattern, most of which on proximal femur such as Gabor filter and wavelet transform [25] and fractal models [11, 17]. Among them, fractal model and image intensity distribution analysis were performed on mandibular trabeculae by Matheus et al. [11]; they noticed that these two algorithms have a strong positive correlation with the detection of osteoporotic changes in some regions of jaws [11]. According to the results, these methods provide high computational complexity among low detection rate.

Local Binary Patterns (LBP) is a non-parametric operator, which describes the local spatial structure and local contrast of an image. For the first time, it was described for graylevel images by Ojala et al. [26]. Until now, LBP has been used for pattern feature extraction in many image processing applications such as defect detection, image retrieval, tumor detection, object tracing, etc. In this paper, we proposed an approach to describe trabecular pattern using modified local binary pattern (MLBP). Trabecular bone analysis with LBP for osteoporosis diagnosis has been performed previously on HIP bone, and until now there is no LBP research on panoramic radiography for detecting osteoporosis patients. Our results show that osteoporosis can be detected using MLBP with high accuracy. Low computational complexity and rotation-invariant are some other advantages of our proposed approach.

\section{Materials and Methods}

To evaluate the performance of our framework, we carried out experiments on a handcaptured dataset, which consists of 138 images of $2400 \times 1200$. The dataset includes two image categories, normal and patient. The normal category consists of 69 jaw panoramic radiography images of 69 different individuals who were without osteoporosis. The pa- 
tient category includes 69 jaw panoramic radiography images of 69 different osteoporotic persons. BMD indices were taken in Shiraz Namazi Hospital and panoramic images were captured in Khojastepour DMFR Center.

\section{Paper Organization}

The paper is organized as follows: In section 2, basic schemas of local binary patterns are discussed, which extract texture features of the input image. Then, modified version of LBP is described to achieve better performance. In section 3, our approach is proposed to analyze jaw panoramic radiography image, and describe trabecular pattern. Section 4 presents the experimental results on osteoporosis detection problem. Finally, the conclusion is presented in Section 5.

\section{Local Binary Patterns}

LBP is an analysis operator which describes the local contrast and local spatial structure of an image. In order to evaluate LBP, at a given pixel position $\left(\mathrm{x}_{\mathrm{c}}, \mathrm{y}_{\mathrm{c}}\right)$, LBP is described as an ordered set of binary comparisons of pixel intensities between the center pixel and its neighbors. Neighborhoods could be assumed circular because of achieving the rotation invariant. LBP is defined as follows:

$$
L B P_{P, R}=\sum_{k=0}^{P-1} \Omega\left(f_{k}-f_{c}\right) 2^{k}
$$

Where:

$$
\Omega(x)= \begin{cases}1 & \text { if } x \geq 0 \\ 0 & \text { else }\end{cases}
$$

$f_{c}$ corresponds to the gray value of the center pixel, and $f_{k}$ to the gray values of the neighbors. $\mathrm{P}$ shows the number of neighbors. Figure 1 shows the process of calculation of LBP code. The $\mathrm{LBP}_{\mathrm{P}, \mathrm{R}}$ operator produces $\left(2^{\mathrm{P}}\right)$ different output values, corresponding to the $2^{\mathrm{P}}$ different binary patterns that can be formed.

When the image is rotated, gray values $f_{p}$ will correspondingly move along the perimeter of the circle around $\mathrm{g}_{\mathrm{c}}$. To remove rotation effect, a unique identifier to each LBP should be assigned as follows:

$$
L B P_{P, R}^{r i}=\min \left\{R O R\left(L B P_{P, R}, i\right) \mid i=0,1, \ldots, P-1\right\}(3)
$$

Where ROR (x, i) performs a circular bitwise right shift on the P-bit number $\mathrm{x}, i$ times.

\section{Modified Local Binary Patterns (MLBP)}

Basic LBP has some disadvantages such as low discrimination and high computational complexity. To solve these problems, Ojala et al. [26] defined a uniformity measure " $U$ ", which corresponds to the number of spatial transitions (bitwise 0/1 changes) in the output pattern. It is shown in Eq. (4). For example, pattern 01001100 has $U$ value of 4 , while 11000001 has $U$ value of 2 .

$U\left(L B P_{P, R}\right)=\left|\Omega\left(f_{0}-f_{c}\right)-\Omega\left(f_{P-1}-f_{c}\right)\right|+\sum_{K=1}^{P-1}\left|\Omega\left(f_{k}-f_{c}\right)-\Omega\left(f_{k-1}-f_{c}\right)\right|$

Patterns with uniformity amount less than $\mathrm{U}_{\mathrm{T}}$ are categorized as uniform patterns. The patterns with uniformity more than $\mathrm{U}_{\mathrm{T}}$ are classified as non-uniforms. Finally, $\mathrm{LBP}_{\mathrm{P}, \mathrm{R}}^{\text {riuT }}$ is computed using Eq. (5).

$L B P_{P, R}^{r i u T}=\left\{\begin{array}{rr}\sum_{k=0}^{P-1} \Omega\left(f_{k}-f_{c}\right) & \text { if } U\left(L B P_{P, R}\right) \leq U_{T} \\ P+1 & \text { elsewhere }\end{array}\right.$

Applying $\mathrm{LBP}_{\mathrm{P}, \mathrm{R}}{ }^{\text {riuT }}$ will assign a label from 0 to $\mathrm{P}$ to uniform patterns and label $\mathrm{P}+1$ to nonuniform patterns. In using $\mathrm{LBP}_{P, R}{ }_{\text {riuT }}$ just one label $(\mathrm{P}+1)$ is assigned to all of the non-uniform patterns. To achieve discriminative features, $\mathrm{U}_{\mathrm{T}}$ should be optimized so that uniform labels cover majority patterns in the image. Experimental results in $[27,28]$ show that if $\mathrm{U}_{\mathrm{T}}$ is selected equal to $(\mathrm{P} / 4)$, only a negligible

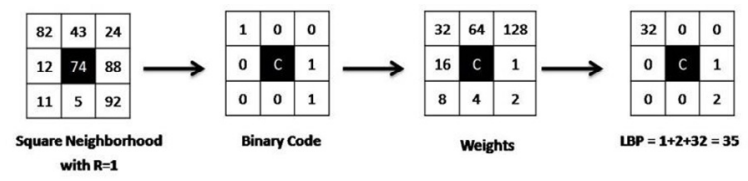

Figure 1: LBP Computing Process 
portion of the patterns in the texture takes label $\mathrm{P}+1$.

$\mathrm{LBP}_{\mathrm{P}, \mathrm{R}}{ }^{\text {riuT }}$ quantifies the occurrence statistics of individual rotation invariant patterns corresponding to certain micro-features in the image; hence, the patterns can be considered as feature detectors. For example, Figure 2 illustrates 36 unique rotation invariant local binary patterns which can occur in the case of $\mathrm{P}=8$ and $R=1$. As it is shown in Figure 2, for instance, pattern $\# 0$ detects bright spots, $\# 8$ dark spots, flat areas, \#4 edges, etc.

\section{Proposed Approach}

As it was described in introduction, the main aim of this paper is to propose an accurate approach for osteoporosis detection using panoramic radiography image analysis. A panoramic radiograph is a panoramic scanning dental X-ray of the upper and lower jaws. It shows a two-dimensional view of a half-circle from ear to ear. Panoramic radiography is a form of tomography, thus, images of multiple planes are taken to make up a composite panoramic image, where maxilla and mandible are in the focal trough and the structures that are superficial and deep to the trough are blurred. An example of panoramic radiogra-

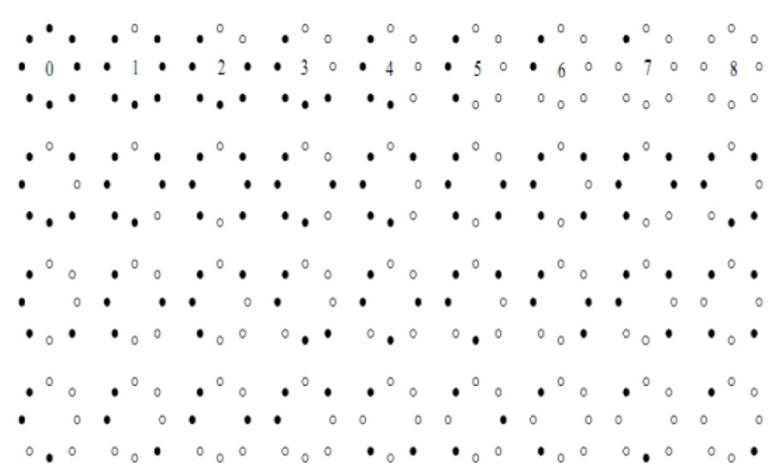

Figure 2: The 36 unique rotation invariant binary patterns that can occur in the circularly symmetric neighbor set of $\mathrm{LBP}_{8, \mathrm{R}}$. Black and white circles correspond to bit values of 0 and 1 in the output phy is shown in Figure 3.

The texture of trabecular patterns in osteoporosis patients is different from non-osteoporosis patients. In this respect, MLBP can be used to define the texture of trabecular pattern. In this respect, first, MLBP should be applied to panoramic radiography image. Then, a feature vector can be computed as follows to describe its trabecular structure.

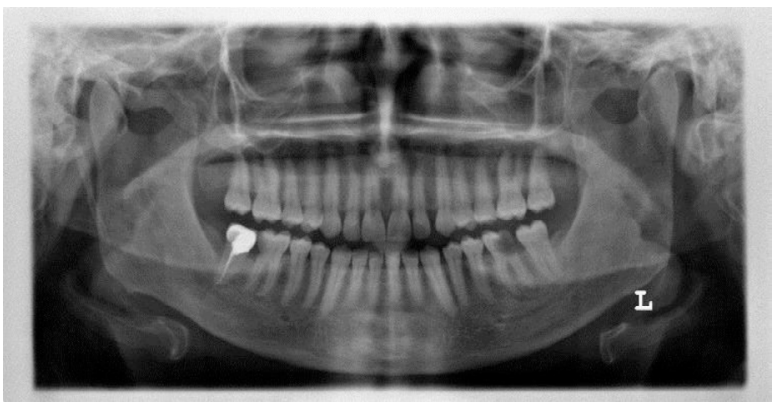

Figure 3: An example of jaw panoramic radiography image

\section{Feature Vector Extraction}

As it was described, a label is assigned to each neighborhood. Regarding Eq. (5), for every image a feature vector $D$ can be extracted which have $P+2$ dimensions as follows:

$$
D=<d_{0}, d_{1}, \ldots, d_{P+1}>
$$

Where:

$$
d_{k}=\frac{N_{k}}{N \times M} \quad 0 \leq k \leq P+1
$$

$N_{k}$ shows a total number of neighbors labeled as $K$. Moreover, $N$ and $M$ are the sizes of the input image where $d_{k}$ is the occurrence probability of label $k$ in whole. To obtain $d_{k}$, first $\mathrm{LBP}_{\mathrm{P}, \mathrm{R}}{ }^{\text {riuT }}$ should be applied on the whole image and the labels are assigned to neighbors. Then, the occurrence probability of each label in the image is regarded as one of the dimensions of the feature vector.

\section{Results}

As it is described in section 2.1, MLBP $_{P, R}$ is a multi-resolution operator which can be 
evaluated using different radius sizes $(\mathrm{R})$ and a number of neighbors $(\mathrm{P})$. In this respect, we evaluated our experiment using $\mathrm{MLBP}_{\mathrm{P}, \mathrm{R}}$ with different radius $\mathrm{R}=1,2$ and 3 . In order to compute the classification accuracy, some efficient classifiers are evaluated based on a 10-fold algorithm as validation such as KNN, Naïve Bayes, J48 tree and voted perceptron. Finally, $\mathrm{KNN}$ with $\mathrm{K}=1$ and $\mathrm{K}=3$, provided maximum accuracy about $99.65 \%$ using $\mathrm{MLBP}_{8,1}$. The results are shown in Table 1. As it is shown, true-positive and false-positive measures are computed. Some jaw panoramic radiography examples of normal and osteoporotic individuals are shown in Figures 4 and 5.

Our osteoporosis diagnosis system was implemented on a personal computer with Core 2Duo CPU with two $2.10 \mathrm{GHz}$ cores and 4.00GB RAM using MATLAB version R2010-B.

\section{Comparison with the State-of-the- art}

To compare the effectiveness of our proposed approach, some state-of-the-art methods in this literature are surveyed as follows. In [29], Zhi et al. proposed an approach for osteoporosis diagnosis using micro-CT images which consisted of two steps. First, the input microCT image is binarized using the basic version of local binary patterns. Next, fractal models and wavelet coefficients are used to extract discriminative features. Reported experimental results in [29] are shown in Table 2. As it is shown in Table 2, the proposed approach provides higher classification accuracy.

In [4], Taguchi et al. proposed an approach for osteoporosis using radiograph jaw images. In [4], two observations are captured from candidates, and then these are compared based on the weighted kappa statistic. In addition, a stepwise multiple regression analysis is evaluated. Reported results of [4] are shown in Table 2.

\section{Computational Complexity}

In order to compute complexity, one of the efficient methods is computing a total number of required texture analysis operations [27, 28]. In our proposed approach, the total number of required operations, which are applied to each test image, are related to the size of image and size of MLBP. If image sample size is considered as $\mathrm{W} \times \mathrm{W}$, the total number of $\mathrm{MLBP}_{\mathrm{P}, \mathrm{R}}$ operations that are applied to each sample are equal to $(\mathrm{W}-2 \mathrm{R}) \times(\mathrm{W}-2 \mathrm{R})$. Where, $\mathrm{R}$ shows the radius of $\mathrm{MLBP}_{\mathrm{P}, \mathrm{R}}$. The total number of required operations for applying $\mathrm{MLBP}_{\mathrm{P}, \mathrm{R}}$ with different $\mathrm{R}$ and $\mathrm{P}$ in a sample dataset image in size of $2400 \times 1200$ are shown in Table 2 . Another way to evaluate computational complexity is computing a total number of extracted feature dimensions. The total number of extracted feature dimensions, which is computed

Table 1: Comparison results (\%) of different classifiers on captured dataset using $\mathrm{MLBP}_{8,1}$

\begin{tabular}{ccccc} 
Validation & Classifier & Accuracy (\%) & TP-Rate & FP-Rate \\
\hline 10Fold & 1NN & 99.65 & 0.996 & 0.004 \\
\hline 10Fold & 3NN & 99.65 & 0.996 & 0.004 \\
\hline 10Fold & $5 N N$ & 98.84 & 0.988 & 0.012 \\
\hline 10Fold & 7NN & 99.31 & 0.993 & 0.008 \\
\hline 10Fold & Naïve Bayes & 94.48 & 0.945 & 0.055 \\
\hline 10Fold & Threshold Selector & 91.03 & 0.91 & 0.081 \\
\hline 10Fold & Voted Perceptron & 54.48 & 0.545 & 0.501 \\
\hline 10Fold & J48 Tree & 96.55 & 0.966 & 0.034
\end{tabular}




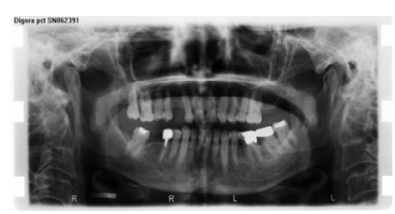

(a)

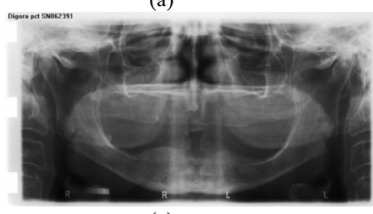

(c)

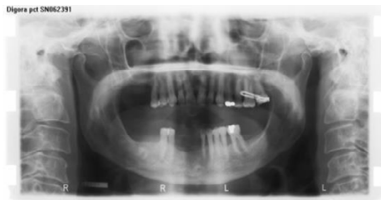

(b)

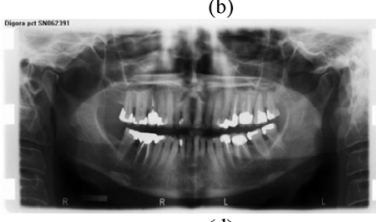

(d)
Figure 4: Some jaw panoramic examples of osteoporotic patients

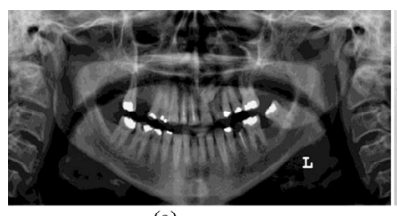

(a)

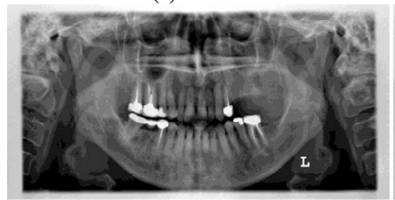

(c)

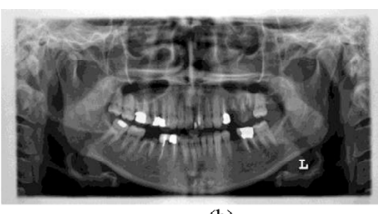

(b)

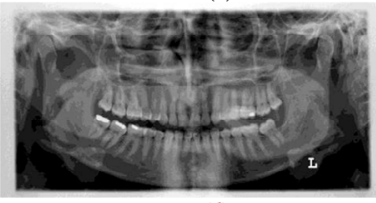

(d)
Figure 5: Some jaw panoramic examples of normal persons without osteoporosis

for each test image, are related just to the size of MLBP $_{P, R}$. The size of extracted feature vectors based on different $\mathrm{MLBP}_{\mathrm{P}, \mathrm{R}}$ operations is shown in Table 3.

\section{Conclusion}

The purpose of this study was to evaluate the relationship between BMD, Jaw trabecular pattern (as one of the main bone strength factors) with image texture analyzing using local binary pattern and osteoporosis in panoramic radiography image. Osteoporosis and fractures are more difficult and costly to treat than to prevent. Therefore, several health care interventions have been proposed to identify those people. $\mathrm{MCW}, \mathrm{M} / \mathrm{M}$ ratio, $\mathrm{PMI}, \mathrm{CI}$, the number of teeth present and FD are the parameters assessed in various studies for detecting their efficacy in screening osteoporosis. We proposed an approach which consists of two steps. First, jaw panoramic radiography image is analyzed using MLBP and a feature vector is extracted. Then, classification is done using a little train set. Results show that proposed approach provides high classification accuracy about 99.65 using $1 \mathrm{NN}$. According to the results, the computational complexity of the proposed method is much lower than previous image analysis methods. Also, the proposed approach is an automatic algorithm which saves more cost in comparison with laboratory approaches.

Rotation invariant, low computational complexity and multi-resolution are some other advantages of our proposed approach.

Table 2: Comparison results (\%) of different methods for osteoporosis classification.

\begin{tabular}{lccc}
\multicolumn{1}{c}{ Method } & Type of Image & Classifier & Accuracy (\%) \\
\hline LBP $_{8,1}+$ Fractal Dimensions [28] & Micro - CT & NBC & 91.5 \\
\hline LBP $_{8,1}+$ Fractal Dimensions [28] & Micro - CT & SVM & 92.6 \\
\hline $\mathrm{LBP}_{8,1}+$ Fractal Dimensions [28] & Micro - CT & KNN & 93.8 \\
\hline $\mathrm{LBP}_{8,1}+$ Wavelet Coefficients [28] & Micro - CT & NBC & 88.3 \\
\hline $\mathrm{LBP}_{8,1}+$ Wavelet Coefficients [28] & Micro-CT & SVM & 87.7 \\
\hline $\mathrm{LBP}_{8,1}+$ Wavelet Coefficients [28] & Micro-CT & KNN & 86.9 \\
\hline Regression [4] & Radiograph Jaw & - & 68.2 \\
\hline Fractal Features + Chi-Square [17] & Radiograph Jaw & KNN & 99 \\
\hline MLBP $_{8,1}$ - Proposed approach & Radiograph Jaw & KNN & 99.65
\end{tabular}


Table 3: The computational complexity of proposed approach in terms of total number required operations and feature vector size.

\begin{tabular}{lcc} 
Operation & $\begin{array}{c}\text { Number of } \\
\text { Required } \\
\text { Operators }\end{array}$ & $\begin{array}{c}\text { Number } \\
\text { of Feature } \\
\text { Dimensions }\end{array}$ \\
\hline $\mathrm{MLBP}_{8,1}$ & 2872804 & 10 \\
\hline $\mathrm{MLBP}_{16,2}$ & 2865616 & 18 \\
\hline $\mathrm{MLBP}_{24,3}$ & 2858436 & 26 \\
\hline $\mathrm{LBP}_{8,1}+$ Fractal [28] & 2872804 & 42 \\
\hline $\mathrm{LBP}_{8,1}+$ Wavelet & 2872804 & 42 \\
Coefficients [28] & &
\end{tabular}

\section{Acknowledgment}

The authors would like to thank the Vicechancellor of Shiraz University of Medical Science for supporting this research (Grant no 9721).

\section{Conflict of Interest}

None

\section{References}

1. Baylink DJ, Strong DD, Mohan S. The diagnosis and treatment of osteoporosis: future prospects. Mol Med Today. 1999;5:133-40. PubMed PMID: 10203737.

2. White S. Oral radiographic predictors of osteoporosis. Dentomaxillofacial radiology. 2002;31:84-92.

3. Dervis E. Oral implications of osteoporosis. Oral Surg Oral Med Oral Pathol Oral Radiol Endod. 2005;100:349-56. doi: 10.1016/j.tripleo.2005.04.010. PubMed PMID: 16122665.

4. Taguchi A, Asano A, Ohtsuka M, Nakamoto T, Suei $\mathrm{Y}$, Tsuda $\mathrm{M}$, et al. Observer performance in diagnosing osteoporosis by dental panoramic radiographs: results from the osteoporosis screening project in dentistry (OSPD). Bone. 2008:43:20913. doi: 10.1016/j.bone.2008.03.014. PubMed PMID: 18482878.

5. Pramudito J, Soegijoko S, Mengko T, Muchtadi F, Wachjudi R. Trabecular pattern analysis of proximal femur radiographs for osteoporosis detection. Journal of Biomedical \& Pharmaceutical Engineering. 2007;1:45-51.

6. Gaur B, Chaudhary A, Wanjari PV, Sunil M, Basavaraj P. Evaluation of panoramic Radiographs as a Screening Tool of Osteoporosis in Post
Menopausal Women: A Cross Sectional Study. J Clin Diagn Res. 2013;7:2051-5. doi: 10.7860/ JCDR/2013/5853.3403. PubMed PMID: 24179941; PubMed Central PMCID: PMC3809680.

7. Johari Khatoonabad M, Aghamohammadzade N, Taghilu H, Esmaeili F, Jabbari Khamnei H. Relationship Among Panoramic Radiography Findings, Biochemical Markers of Bone Turnover and Hip BMD in the Diagnosis of Postmenopausal Osteoporosis. Iran J Radiol. 2011;8:23-8. PubMed PMID: 23329912; PubMed Central PMCID: PMC3522411.

8. Horner K, Devlin H. The relationship between mandibular bone mineral density and panoramic radiographic measurements. J Dent. 1998;26:337-43. PubMed PMID: 9611939.

9. Taguchi A, Suei Y, Ohtsuka M, Otani K, Tanimoto $\mathrm{K}$, Ohtaki M. Usefulness of panoramic radiography in the diagnosis of postmenopausal osteoporosis in women. Width and morphology of inferior cortex of the mandible. Dentomaxillofac Radiol. 1996;25:263-7. doi: 10.1259/dmfr.25.5.9161180. PubMed PMID: 9161180.

10. Kanis JA, Johnell 0. Requirements for DXA for the management of osteoporosis in Europe. Osteoporos Int. 2005;16:229-38. doi: 10.1007/s00198004-1811-2. PubMed PMID: 15618996.

11. Oliveira ML, Pedrosa EF, Cruz AD, Haiter-Neto F, Paula FJ, Watanabe PC. Relationship between bone mineral density and trabecular bone pattern in postmenopausal osteoporotic Brazilian women. Clin Oral Investig. 2013;17:1847-53. doi: 10.1007/ s00784-012-0882-2. PubMed PMID: 23239088.

12. Roberts MG, Graham J, Devlin H. Image texture in dental panoramic radiographs as a potential biomarker of osteoporosis. IEEE Trans Biomed Eng. 2013;60:2384-92. doi: 10.1109/ TBME.2013.2256908. PubMed PMID: 23568478.

13. Khojastehpour L, Mogharrabi S, Dabbaghmanesh $\mathrm{MH}$, Iraji Nasrabadi N. Comparison of the mandibular bone densitometry measurement between normal, osteopenic and osteoporotic postmenopausal women. J Dent (Tehran). 2013;10:203-9. PubMed PMID: 25512746; PubMed Central PMCID: PMC4264091.

14. Yasar F, Akgunlu F. Evaluating mandibular cortical index quantitatively. Eur J Dent. 2008;2:283-90. PubMed PMID: 19212535; PubMed Central PMCID: PMC2634783.

15. Khojastehpour L, Shahidi S, Barghan S, Aflaki E. Efficacy of panoramic mandibular index in diagnosing osteoporosis in women. J Dent (Tehran). 2009;6:11-5.

16. Hildebolt C. Osteoporosis and oral bone loss. Den- 


\section{tomaxillofacial Radiology. 1997;26:3-15.}

17. Yasar F, Akgunlu F. The differences in panoramic mandibular indices and fractal dimension between patients with and without spinal osteoporosis. Dentomaxillofac Radiol. 2006;35:1-9. doi: 10.1259/ dmfr/97652136. PubMed PMID: 16421256.

18. Mohajery M, Brooks SL. Oral radiographs in the detection of early signs of osteoporosis. Oral Surg Oral Med Oral Pathol. 1992;73:112-7. PubMed PMID: 1603549.

19. Peitgen $H-0$, Jürgens $H$, Saupe D. Introduction to fractals and chaos: Springer-Verlag; 1992.

20. Bollen AM, Taguchi A, Hujoel PP, Hollender LG. Case-control study on self-reported osteoporotic fractures and mandibular cortical bone. Oral Surg Oral Med Oral Pathol Oral Radiol Endod. 2000;90:518-24. doi: 10.1067/moe.2000.107802. PubMed PMID: 11027391.

21. Klemetti E, Kolmakov S, Kröger H. Pantomography in assessment of the osteoporosis risk group. Eur J Oral Sci. 1994;102:68-72.

22. Geraets WG, Van der Stelt PF, Netelenbos CJ, Elders PJ. A new method for automatic recognition of the radiographic trabecular pattern. J Bone Miner Res. 1990;5:227-33. PubMed PMID: 2333781.

23. Link TM, Majumdar S, Lin JC, Augat P, Gould RG, Newitt $D$, et al. Assessment of trabecular structure using high resolution CT images and texture analysis. J Comput Assist Tomogr. 1998;22:15-24. PubMed PMID: 9448755.

24. Shahidi S, Bahrampour E, Soltanimehr E, Zamani A, Oshagh M, Moattari M, et al. The accuracy of a designed software for automated localization of craniofacial landmarks on CBCT images. BMC Med Imaging. 2014;14:32.

25. Houam L, Hafiane A, Boukrouche A, Lespessailles $E$, Jennane R, editors. Texture characterization using local binary pattern and wavelets. Application to bone radiographs. Image Processing Theory, Tools and Applications (IPTA), 2012 3rd International Conference on; 2012: IEEE.

26. Ojala T, Pietikainen M, Maenpaa T. Multiresolution gray-scale and rotation invariant texture classification with local binary patterns. IEEE Trans Pattern Anal Mach Intell. 2002;24:971-87.

27. Fekri-Ershad S, Tajeripour F. A robust approach for surface defect detection based on one dimensional local binary patterns. Indian Journal of Science and Technology. 2012;5:3197-203.

28. Tajeripour F, Fekri-Ershad S. Developing a novel approach for stone porosity computing using modified local binary patterns and single scale retinex. Arabian Journal for Science and Engineering. 2014;39:875-89.

29. Gao Z, Hong W, Xu Y. Trabecular bone microCT images analysis for osteoporosis diagnosis. $J$ Comput Inf Syst. 2012;8:10341-7. 